\title{
Biomedical engineering
}

\section{Radio-frequency ring applicator: energy distributions measured in the CDRH phantom}

\author{
G. C. van Rhoon'
}

P. Raskmark ${ }^{2}$

P. M. van den Berg

S. N. Hornsleth ${ }^{2}$

'Department of Hyperthermia, Dr. Daniel den Hoed Cancer Center, PO Box 5201, 3008 AE, Rotterdam, The Netherlands

2 Institute for Electronic Systems, University of Aalborg, Aalborg, Denmark

${ }^{3}$ Laboratory of Electromagnetic Research, Delft University of Technology, Delft, The Netherlands

\begin{abstract}
SAR distributions were measured in the $C D R H$ phanton, a $1 \mathrm{~cm}$ fatequivalent shell filled with an abdomen-equivalent liquid $\left(\sigma=0.4-1.0 \mathrm{~S} \mathrm{~m}^{-1}\right.$; dimensions $22 \times 32 \times 57 \mathrm{~cm}$ ) to demonstrate the feasibility of the ring applicator to obtain deep heating. The ring electrodes were fixed in a PVC tube; diameter $48 \mathrm{~cm}$, ring width $20 \mathrm{~cm}$ and gap width between both rings $31.6 \mathrm{~cm}$. Radio-frequency energy was fed to the electrodes at eight points. The medium between the electrodes and the phantom was deionised water. The SAR distribution in the liquid tissue volume was obtained by a scanning E-field probe measuring the E-field in all three directions. With equal amplitude and phase applied to all feeding points, a uniform SAR distribution was measured in the central cross-section at $30 \mathrm{MHz}$. With RF energy supplied to only four adjacent feeding points lothers were connected to a 50 $\Omega$ load), the feasibility to perform amplitude steering was demonstrated; SAR values above $50 \%$ of the maximum SAR were measured in one quadrant only. SAR distributions obtained at $70 \mathrm{MHz}$ showed an improved focusing ability; a maximum at the centre exists for an electric conductivity of the abdomen-equivalent tissue of 0.6 and $0.4 \mathrm{~S} \mathrm{~m}^{-1}$.
\end{abstract}

Keywords-Circumferential E-field, Loco-regional deep hyperthermia, Phase and amplutide control, Radio-frequency, Ring applicator, SAR distribution, SAR steering

Med. \& Biol. Eng. \& Comput., 1994, 32, 643-648

\section{Introduction}

FOR LOCO-REGIONAL hyperthermia, several non-invasive electromagnetic (EM) devices (DE LEEUW and LAGENDIJK, 1987; FranCONI, 1987; VISSER et al., 1987; HEINZL et al., 1990; TURNER, et al. 1989) have been developed using either quasi-static or radiative methods to transfer the energy to the patient. All of these are unable to deposit the power selectively in the tumour. Clinical experience with loco-regional hyperthermia has shown that therapeutic temperatures can be achieved in deep-seated tumours, but also indicated the difficulty in fulfilling the time-temperature goals in pelvic tumours (KAPP et al., 1988; SAPOZINK et al., 1988; SHIMM et al., 1988).

To optimise the specific absorption rate (SAR) and the temperature distribution for patient size and tumour location, most of the radiative hyperthermia systems provide SAR steering by phase and amplitude, and frequency variation. Despite these essential features (STrOHBEHN et al., 1989; WUST et al., 1991), the magnitude of the power application during clinical treatment is often limited by hot spots produced at bony prominences of the

Reprint requests should be addressed to $G$. C. van Rhoon First received 21 January and in final form 23 August 1993 (C) IFMBE: 1994 pelvis, perineal fatty tissue and anterolateral thigh areas (Feldman et al., 1991; Oleson*).

These findings emphasise the need for continuous applicator development, with the aim of improving the flexibility and adaptability of the SAR distribution to the patient's anatomy. The ring applicator developed in our institute and by Franconi (FRANCONI, 1987) may offer a solution to some of these problems. As described elsewhere (VAN RHOON, 1988, 1990; RAGANELLA, 1989), the ring applicator consists of two thin ring-shaped electrodes, placed around the volume to be heated at the upper and lower margin. With large diameters of the ring electrodes, coupling the electrodes to the patient through water and at frequencies above $25 \mathrm{MHz}$ a radiative circumferential E-field distribution is created around the phantom. As with other radiative systems, like the coaxial TEM applicator (DE LEEUW et al., 1990), the Sigma applicator (TURNER et al., 1989) or the four waveguide system (VAN DIJK et al., 1990), constructive interference occurs and may result in maximum energy deposition at the centre of the phantom.

In earlier work, we have demonstrated, both experimentally and theoretically, the feasibility of the ring applicator in controlling the longitudinal length of the treatment volume and the intensity of the near field, in small

\footnotetext{
- Oleson, personal communication, 1990
} 


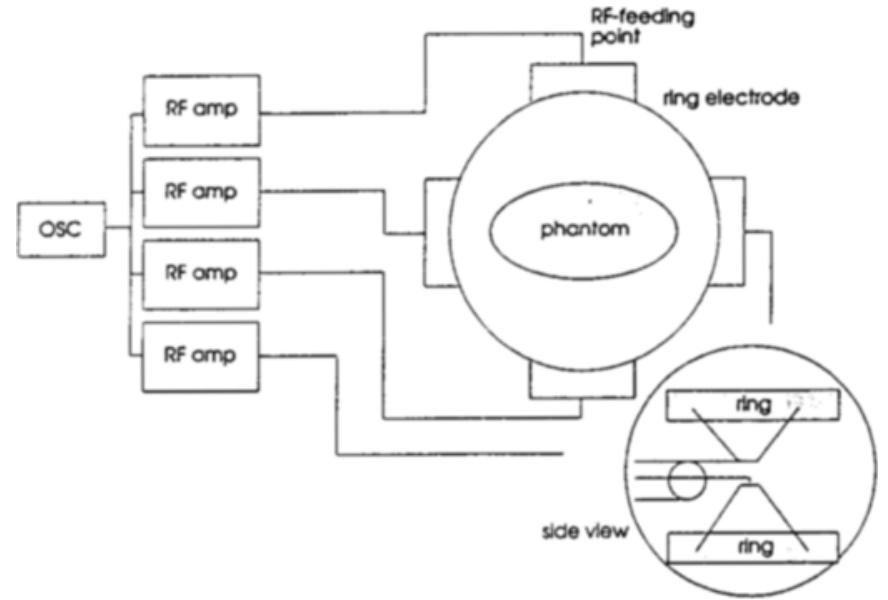

Fig. 1 Schematic representation of the experimental set-up: $R F$ amplifiers and connection of the RF-feeding points

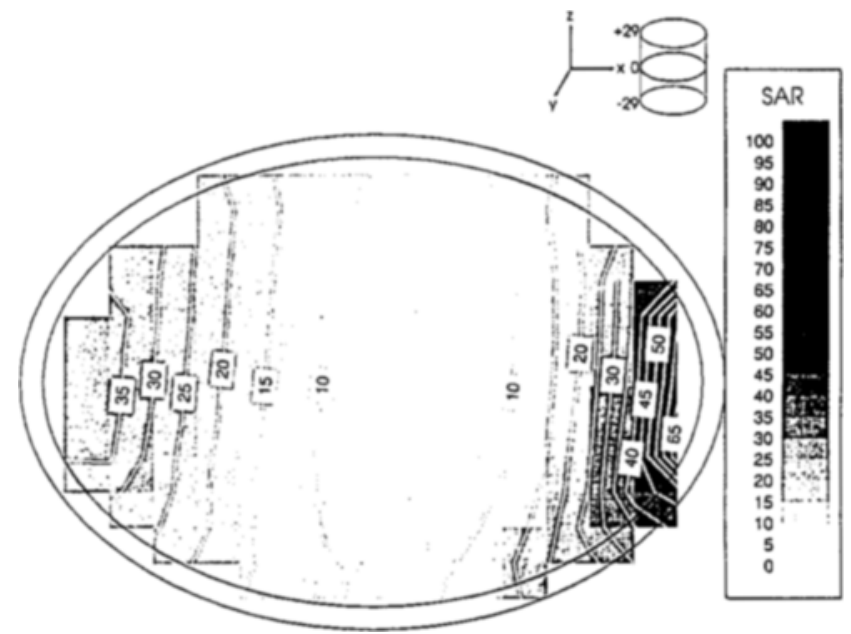

Fig. 2 Relative SAR distribution measured at the cross-sectional. mid-plane of the phantom and the ring electrodes; one $R F$-feeding point at the right side; frequency $33 \mathrm{MHz}$; $\sigma=0.6 \mathrm{Sm}^{-1}$

cylindrical phantoms (diameter $\leq 13.5 \mathrm{~cm}$ ) by changing the applicator configuration. These studies were performed for a frequency of $27.12 \mathrm{MHz}$. More recent theoretical studies by our group $\dagger$ predict that these features of the ring applicator remain available in large phantoms and over a broad frequency range $(30-90 \mathrm{MHz})$. Therefore, the ring applicator might possess an improved ability to reduce the number of hot spots or to shift the locations of hot spots.

This paper reports our experimental work to demonstrate the feasibility of the ring applicator in obtaining deep heating in a phantom of realistic size, i.e. the well known CDRH phantom developed by the Centre for Devices and Radiological Health (CDRH).

\section{Materials and methods}

The applicator consists of a PVC tube (length $58 \mathrm{~cm}$, inner diameter $48 \mathrm{~cm}$ ) in which the ring electrodes of tin-plate copper (thickness $0.2 \mathrm{~mm}$ ) were glued to the inner surface. The electrodes had a diameter of $48 \mathrm{~cm}$, width of $10 \mathrm{~cm}$, and the gap width between the upper and lower ring electrode was $31.6 \mathrm{~cm}$. The medium between the electrodes

TVAN RHOON, G. C. (1991): 'Development of a ring applicator for deep pelvic heating with the ability to control the longitudinal length of the treatment volume'. Grant application to the Dutch Cancer Society

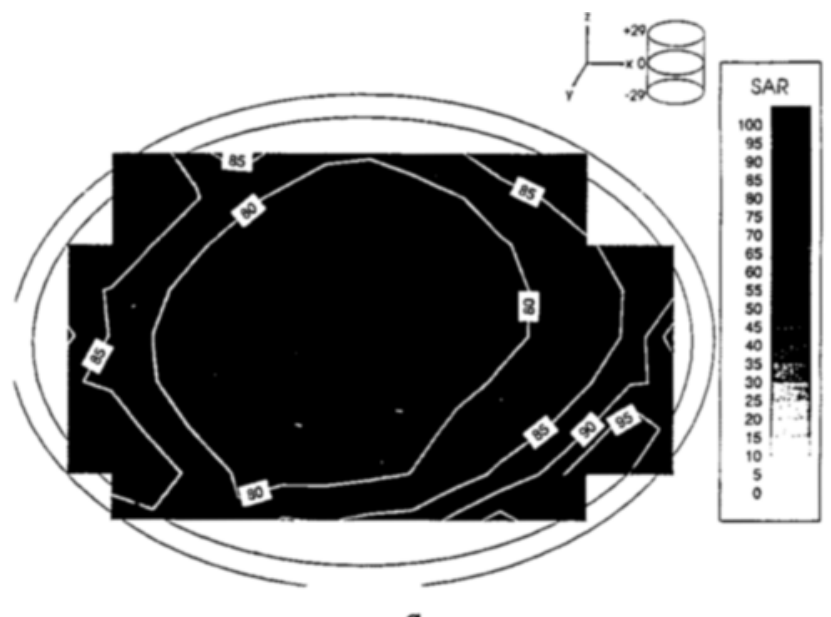

a

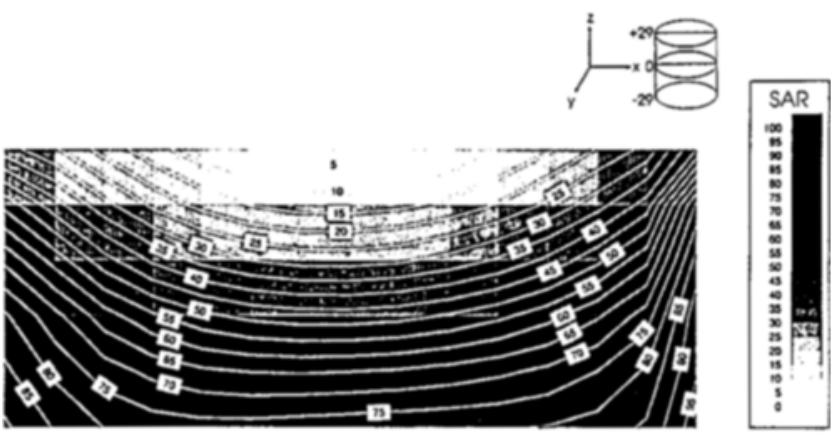

b

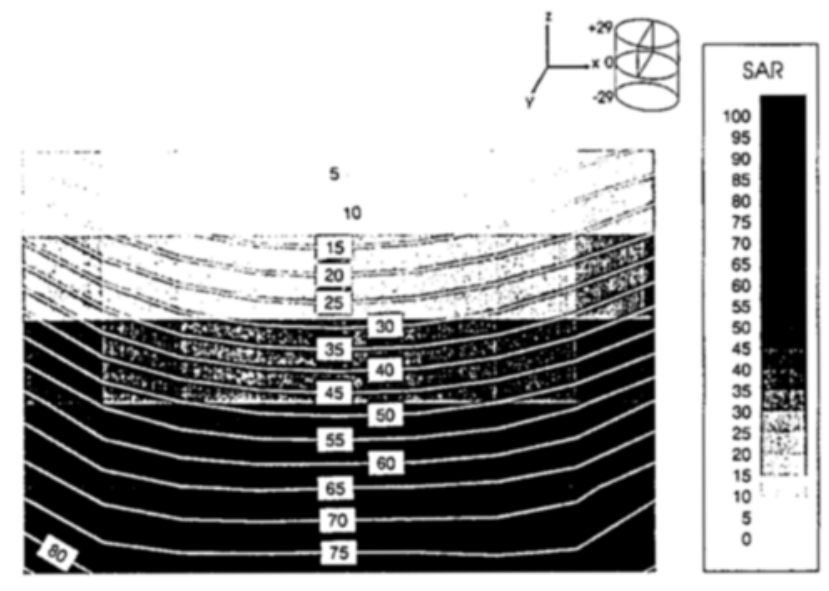

c

Fig. 3 (a) Relative SAR distribution measured at the crosssectional mid-plane of the phantom and the electrodes; (b) in the $x z ;(c)$ yz planes; eight $R F$-feeding points; frequency $33 \mathrm{MHz} ; \sigma=0.6 \mathrm{Sm}^{-1}$

and the phantom is deionised water. Radio-frequency (RF) energy was fed to the ring applicator at one, four or eight different points to investigate the effects of potential distribution over the electrode surface (Fig. 1). A home-built $\dagger$ four-channel ( $50 \mathrm{~W}$ per channel) broadband RF power generator was used to drive each feeding point. Amplitude and phase of each channel could be controlled independently. RF power per channel was measured by Bird in-line power meters, and phase per channel was measured through a bidirectional coupler with a Hewlett Packard vector-voltmeter§. Open-line transformers were used to match the impedance at each feeding point of the ring applicator. Experiments were performed at frequencies. of 33 and $70 \mathrm{MHz}$.

† Institute for Electronic Systems \$ HP 8405A 


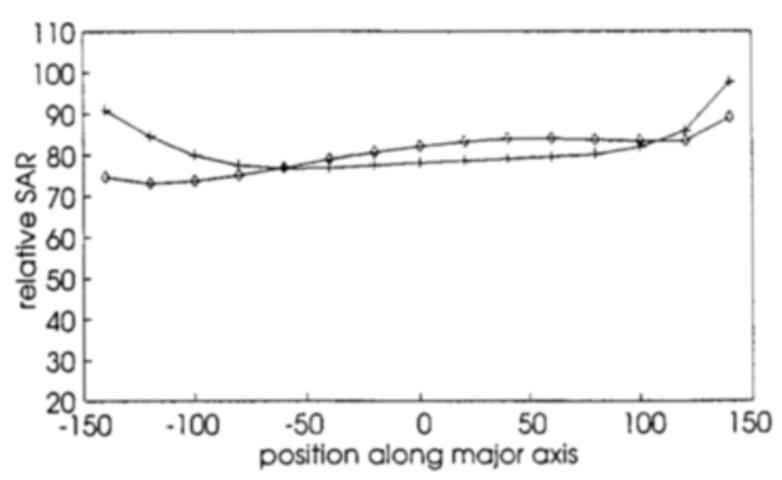

- sigma $0.4 \mathrm{~S} \mathrm{~m}^{-1}+\operatorname{sigma} 0.6 \mathrm{~S} \mathrm{~m}^{-1}$

a

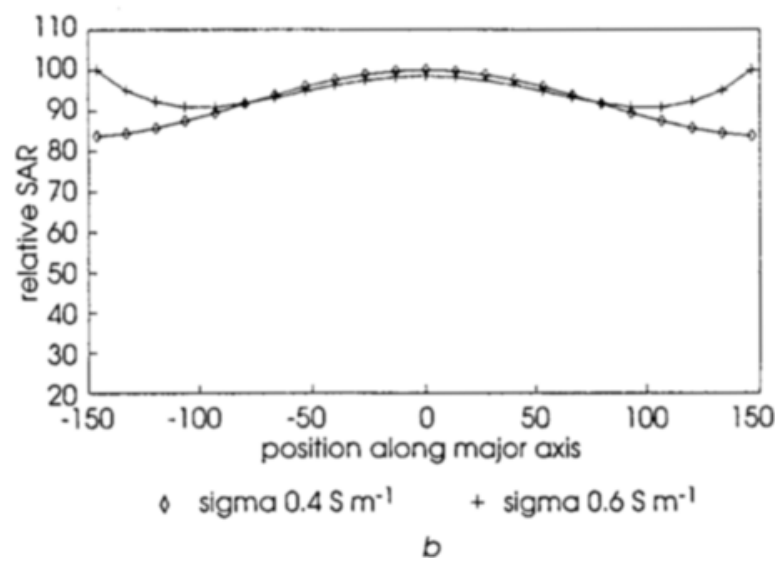

Fig. 4 Comparison of the (a) measured and (b) predicted $S A R$ profile along the major axis of the phantom at the central cross-section; frequency $33 \mathrm{MHz} ; \sigma=0.4$ and $0.6 \mathrm{~S} \mathrm{~m}^{-1}$

The CDRH phantom consists of a $1 \mathrm{~cm}$ thick, solid fat-equivalent shell filled with abdomen-equivalent liquid, with an elliptical cross-section of $22 \times 32 \mathrm{~cm}$, and is $57 \mathrm{~cm}$ long. Various electric conductivities $\left(\sigma=0.4-1.0 \mathrm{~S} \mathrm{~m}^{-1}\right.$ at $20^{\circ} \mathrm{C}$ ) of the abdomen-equivalent liquid were obtained by solving different amounts of $\mathrm{NaCl}\left(2,3\right.$ or $\left.5 \mathrm{~g} \mathrm{l}^{-1}\right)$ in tap water, based on Stogryn's (STOGRYN, 1971) formula. This allowed us to investigate the focusing ability of the ring applicator as a function of conductivity. The SAR distribution was determined with a three-dimensional E-field measuring system developed at the Institute of Electronic Systems (RASKMARK and Gross, 1987). The system uses a single Schottky diode** electric (E) field dipole probe (length $8 \mathrm{~mm}$ ) connected to a high-impedance DC voltmeter via a pair of twisted high-resistance leads $\dagger \dagger$. The three orthogonal E-field components are measured by orienting the dipole at an angle of $54.7^{\circ}$ to the axis of the probe. Three rotations of the probe over $120^{\circ}$ can resolve the entire E-field. A relative calibration of the probe is performed in the media of interest, and the magnitude of all three field components is measured. Scanning is performed only in the inner elliptical compartment and is accomplished in three dimensions with a Hewlett Packard plotter mounted on a stepper motor-controlled platform. Spatial accuracy within a horizontal plane $(x, y)$ is better than $\pm 0.03 \mathrm{~mm}$ and for the z-direction $\pm 1 \mathrm{~mm}$. The overall accuracy for the E-field measurements is $\pm 5 \%$ for signals over a $20 \mathrm{~dB}$ range. The time to perform a scan over half the CDRH phantom with a $20 \mathrm{~mm}$ resolution $(>5000$ pixels) is approximately 4 hours.

In addition, we used a theoretical model to compute the electromagnetic field distribution induced by the ring

\footnotetext{
* HP5082-2774
}

t† Flurosint $819,250 \mathrm{k} \Omega . \mathrm{ft}$

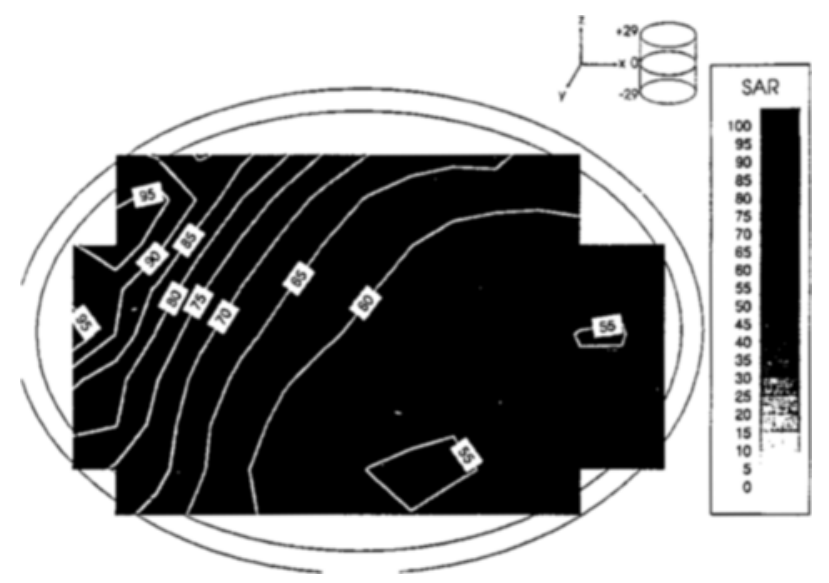

Fig. 5 SAR distribution measured at the central cross-section with equal power output from the upper and left $R F$-feeding point, whereas the lower and right $R F$-feeding points have zero power output; frequency $33 \mathrm{MHz} ; 0.6 \mathrm{~S} \mathrm{~m}^{-1}$

applicator in radially layered cylindrical tissue configurations. The abdomen-equivalent tissue cylinder (radius $15 \mathrm{~cm}$ ) was analogue to the CDRH phantom, enclosed by a fat layer of $1 \mathrm{~cm}$ thickness and assumed to be indefinitely long. Furthermore, the model assumes that the pair of coaxially mounted ring electrodes are perfect conductors, infinitely thin and that the potential is constant over the perimeter of the ring electrodes. For a detailed description see work by Sowinski et al. (SOWINSKI et al., 1989).

\section{Results}

\subsection{RF-feeding points}

Several experiments were performed to investigate the influence of the frequency and the number and location of the energy feeding points on the resulting SAR distribution. Fig. 2 shows the SAR distribution measured at the cross-sectional mid-plane between the electrodes when the ring applicator is operated at $33 \mathrm{MHz}$, using a single RF-feeding point. The relative SAR distribution shows high energy absorption at the side of the RF-feeding point, zero energy absorption at the centre of the phantom, and a second mirror-like image, high energy absorption area at the side opposite to the RF-feeding point. Maximum energy absorption with this set-up was measured in front of the inner edge of the upper ring electrode at the side opposite to the RF-feeding point. The mirror-like image high SAR area can be explained by the induced potential distribution over the perimeter of the electrode. At $33 \mathrm{MHz}$, the half wavelength of the EM field in water $\left(30^{\circ} \mathrm{C}\right)$ is $\pm 53 \mathrm{~cm}$ and the half perimeter of the electrode is $75 \mathrm{~cm}$. This means that the EM field at the side opposite to the RF-feeding point is out of phase with the EM field at the RF-feeding point. As a consequence, the circumferential E-field distribution induces a destructive interference at the centre of the phantom. Operating the applicator with four RF-feeding points resulted in some improvement at $33 \mathrm{MHz}$ but not at $70 \mathrm{MHz}$. With regard to constructive interference at the centre of the phantom, stray radiation and control of the RF-power to each channel, the best results were obtained when eight RF-feeding points were used. The increase to eight RF-feeding points was realised by splitting the RF-signal of a single amplifier over two RF-feeding points. In addition, the experiments performed at $70 \mathrm{MHz}$ demonstrated a better operating of the ring applicator system when the RF-feeding points were located symmetrically with regard to the phantom (Fig. 1). In all experiments reported hereafter, eight RF-feeding points have been used. 

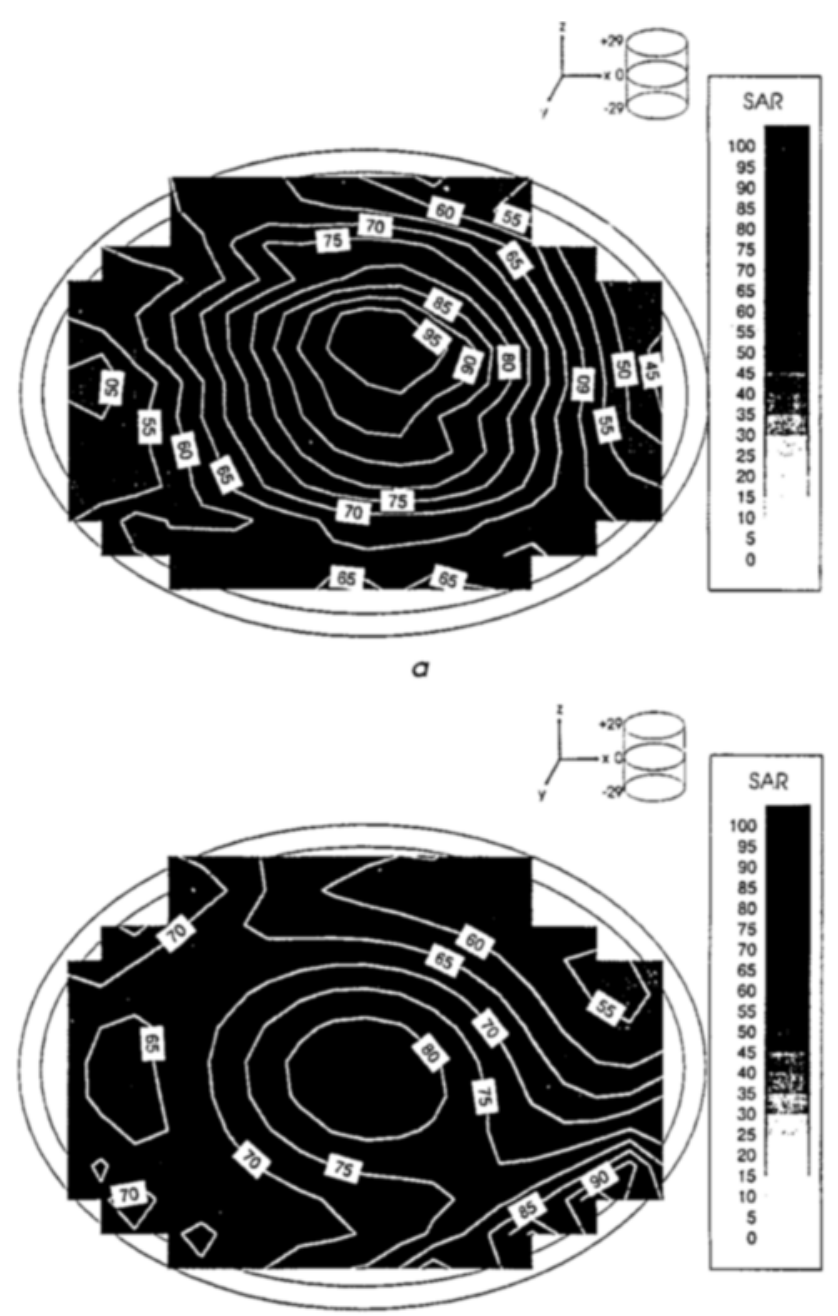

b

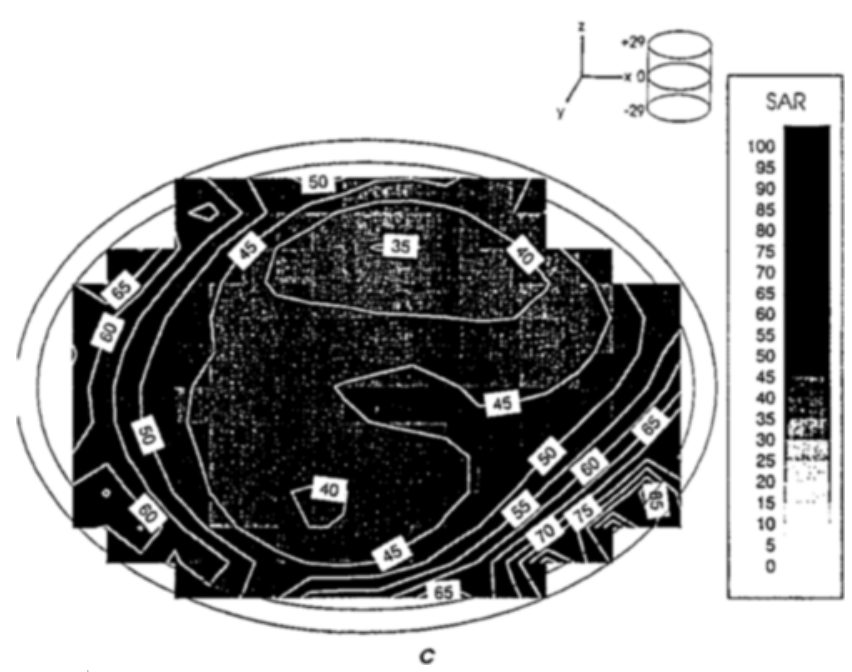

Fig. 6 SAR distributions measured at the central cross-section for (a) $\sigma=0.4$; (b) 0.6 ; (c) $1.0 \mathrm{~S} \mathrm{~m}^{-1}$; frequency $70 \mathrm{MHz}$

\section{$3.233 \mathrm{MHz} S A R$ experiments}

The ability of the ring applicator to produce heat at depth within the CDRH phantom is illustrated in Figs. $3 a-c$. Fig. $3 a$ shows the SAR distribution at the central cross-section, with $\sigma=0.6 \mathrm{~S} \mathrm{~m}^{-1}$ for the inner phantom part. The SAR distribution in the $\mathrm{xz}$ and $\mathrm{yz}$ plane is shown in Figs. $3 b$ and c. As can be seen from Fig. $3 a$, the SAR distribution at the central cross-section is more or less uniform. The latter is in good agreement with the predicted SAR distribution for the cylindrical model, with an overall diameter of $32 \mathrm{~cm}$
(Fig. 4). The small hot spots, including the maximum SAR value, in the lower right-hand corner of the SAR distribution are caused by an imperfection in the experimental set-up.

The SAR distribution measured with $\sigma=0.4 \mathrm{~S} \mathrm{~m}^{-1}$ for the inner phantom part did not differ significantly from the SAR distribution measured with $\sigma=0.6 \mathrm{~S} \mathrm{~m}^{-1}$. Fig. $4 a$ shows the measured SAR profile along the major axis of the phantom at the central cross-section for both conductivities. For comparison, the SAR profile along the axis at the central cross-section as predicted for the cylindrical model is shown in Fig. $4 b$. Reasonable agreement exists, although the measured SAR profiles do not show the focusing ability as might have been expected from the predicted SAR profiles. This difference might be due to the fact that with the cylindrical model maximum constructive interference occurs, whereas with the elliptical phantom the differences in path length between the major and minor axis results in a non-optimal interference.

Fig. 5 shows the SAR distribution measured at the central cross-section with power output from only two of the four amplifiers. Equal power was applied to the left and upper RF-feeding points and zero power was applied to the right and lower RF-feeding points. Note, however, that these $\mathrm{RF}$-feeding points remained connected to the amplifiers, which resembled a $50 \Omega$ load. As shown in Fig. 5, this amplitude steering resulted in maximum SAR values in the upper left quadrant. In the case that the right and lower two RF-feeding points were disconnected from the amplifiers, which act as an open line, a much less favourable SAR distribution was measured. As before, the EM-fields originating from the active $\mathrm{RF}$-feeding points were reflected $180^{\circ}$ out of phase at the open RF-feeding points. As a result, destructive interference occurred at the centre and high SAR values were measured at the periphery of the phantom.

\section{3 $70 \mathrm{MHz} S A R$ experiments}

At this frequency, the work was concentrated on SAR experiments to demonstrate the focusing ability of the ring applicator. In all experiments, equal power was applied to each RF-feeding point. The SAR distributions measured at the central cross-section for $\sigma=0.4,0.6$ and $1.0 \mathrm{~S} \mathrm{~m}^{-1}$ are shown in Figs. $6 a-c$, respectively. No focusing effect was found with $\sigma=1.0 \mathrm{~S} \mathrm{~m}^{-1}$ (muscle-equivalent). Lowering $\sigma$ to $0.6 \mathrm{~S} \mathrm{~m}^{-1}$, a value often quoted as being equivalent to the average electric conductivity of all abdominal tissues at this frequency, results in a small but definitive maximum at the centre. If $\sigma$ is decreased to $0.4 \mathrm{~S} \mathrm{~m}^{-1}$, the resulting SAR distribution shows, as expected, a more pronounced focusing with the maximum SAR at the centre of the phantom. Here again, the small hot spots, including the maximum SAR value, in the lower right-hand corner of the SAR distributions are caused by an imperfection in the experimental set-up. Figs. $7 a$ and $b$ show that good agreement exists between the measured SAR profile along the major axis at the central cross-section of the elliptical phantom and the SAR profile along the axis at the central cross-section predicted for the cylindrical model.

\section{Discussion}

To obtain maximum constructive interference at the centre of the phantom, it is essential to create a circumferential E-field distribution with constant phase and amplitude around the phantom. Whether such a circumferential E-field distribution can be obtained strongly depends on the diameter of the ring applicator, the operating frequency, 
and the number and location of the RF-feeding points. For the ring applicator configuration, as used in this study, we found that with eight RF-feeding points a circumferential E-field distribution could be created with a good constructive interference at the centre of the phantom. This finding is in agreement with the Sigma-60 applicator\$\&, which uses the same number of dipole applicators.

The hot spots, including the maximum SAR value, as measured in the lower right-hand corner of several SAR distributions (Figs. $3 a, 6 b$ and $c$ ) are caused by an imperfection in the experimental set-up. This was experimentally confirmed at $70 \mathrm{MHz}$ with three different set-up combinations of the phantom and the applicator. The standard applicator and phantom set-up served as a reference for this experiment, with power settings per channel such that a good symmetrical SAR distribution (Fig. 6a) was obtained. Turning only the CDRH phantom by $180^{\circ}$ and using the same amplitude settings, no difference was found in the measured SAR distribution. However, turning the whole applicator set-up (including the phantom) by $180^{\circ}$ resulted in a SAR distribution with hot spots and the maximum SAR in the lower right-hand corner. The latter indicates that part of the EM-field from the applicator is coupled to the surrounding experimental set-up, whereby the platform (large metallic parts) to support the HP plotter in particular is suspected of having an influence on the EM-field.

The SAR distribution measured for the ring applicator at $70 \mathrm{MHz}$ with the lower values of electric conductivity $\left(0.4\right.$ and $\left.0.6 \mathrm{~S} \mathrm{~m}^{-1}\right)$ are in good agreement with those reported for the Sigma-60 dipole applicator (TURNER et al., 1989) and the Coaxial TEM applicator (DE LEEUw et al., 1991). For all three systems, the SAR distribution is characterised by a broad maximum at the centre of the phantom and minimum SAR values at a distance of approximately $10 \mathrm{~cm}$ from the centre. The SAR distribution measured for $\sigma=1.0 \mathrm{~S} \mathrm{~m}^{-1}$, muscle-equivalent at $70 \mathrm{MHz}$, demonstrated the effect of the reduced penetration depth with increasing electric conductivities. As expected, the radiative $E M$ fields are rapidly absorbed, showing maximum SAR values at the periphery of the phantom, and the constructive interference is no longer able to create a local maximum.

With the lower frequency of $33 \mathrm{MHz}$, a better penetration depth exists. However, the long wavelength prohibits focusing to a small volume. As expected, the measured SAR profile is indeed characterised by a more or less uniform distribution in reasonable agreement with theoretical predictions. The non-symmetrical distribution is next to the experimental set-up, also a consequence of the sampling method and the normalisation to the maximum value measured. The ability of the ring applicator to perform SAR steering by amplitude control has been demonstrated experimentally. However, as there is no focus at a frequency of $33 \mathrm{MHz}$, it is clear that SAR steering is limited and can only result in directing the major energy absorption to one quadrant of the phantom. Nevertheless, the results are encouraging with regard to the capability of the ring applicator to perform SAR steering at the higher frequencies $(70-100 \mathrm{MHz})$.

The solid material of the fat-equivalent material prohibited the measurement of the SAR within the fat layer. However, the direction of the E-field of the ring applicator is mainly parallel to the body-axis, and thus parallel to the fat-muscle interface, and low energy absorption in the fatty tissue can be expected. In this respect, the ring applicator does not differ from the other existing radiative systems for

developed by BSD Corporation

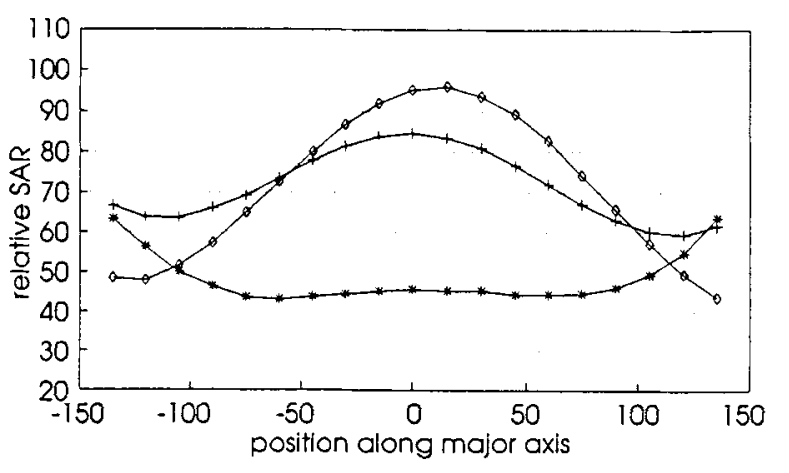

o sigma $0.4 \mathrm{~S} \mathrm{~m}^{-1}+\operatorname{sigma~} 0.6 \mathrm{~S} \mathrm{~m}^{-1} *$ sigma $1.0 \mathrm{~S} \mathrm{~m}^{-1}$

a

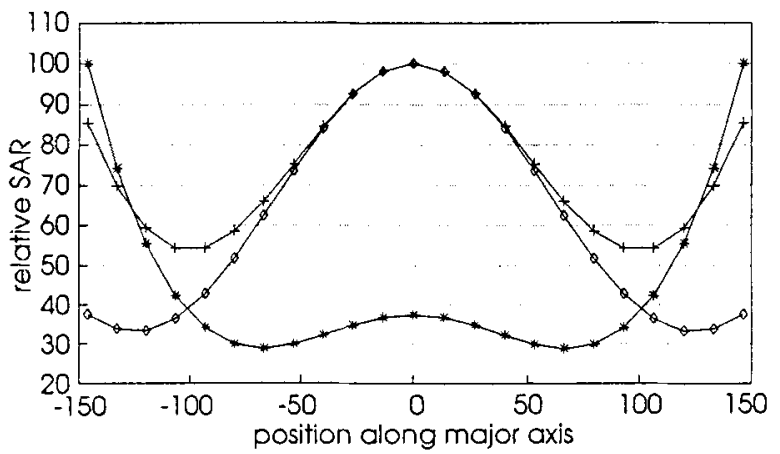

o sigma $0.4 \mathrm{~S} \mathrm{~m}^{-1}$ + sigma $0.6 \mathrm{~S} \mathrm{~m}^{-1}$ * sigma $1.0 \mathrm{~S} \mathrm{~m}^{-1}$

b

Fig. 7 Comparison of the (a) measured and (b) predicted $S A R$ profile along the major axis of the phantom at the central cross-section; frequency $70 \mathrm{MHz} ; \sigma=0.4,0.6$ and $1.0 S \mathrm{~m}^{-1}$

loco-regional deep hyperthermia. The theoretic modelling performed does indeed predict a low SAR in the fatty tissue for all experimental set-ups. The ratio of maximum SAR in the fatty tissue, located below the inner edge of the ring electrodes, to the maximum SAR in the abdomen equivalent tissue was at $33 \mathrm{MHz} 0.7$ and 0.85 for $\sigma=0.4$ and $0.6 \mathrm{~S} \mathrm{~m}^{-1}$, respectively. At $70 \mathrm{MHz}$, these ratios were 0.35 , 0.40 and 0.35 for $\sigma=0.4,0.6$ and $1.0 \mathrm{~S} \mathrm{~m}^{-1}$, respectively. Furthermore, by adapting the configuration of the ring applicator (VAN RHOON et al., 1990) it is possible to optimise the SAR distribution such that a minimal heating of the fat tissue exists in front of the ring electrodes.

With regard to the ability to obtain heating at depth, there seems to be no difference between the various radiative hyperthermia systems for loco-regional deep heating. This has been confirmed elsewhere by Schneider and Van Dijk (SCHNEIDER and VAN DIJK, 1991), who used the LED matrix, to compare the SAR distribution at the central cross-sectional plane of three loco-regional deep heating systems and found no difference in the SAR characteristics. The latter does not exclude the possibility that a specific design of the deep heating system might offer particular advances for the clinical application of loco-regional hyperthermia. With the Coaxial TEM applicator for example, the open water bolus results in a significant reduction of water pressure on the abdomen of the patient. An advantage of the ring applicator, in addition to the theoretical feasibility of performing longitudinal SAR control, might be that the gap between the ring electrodes allows the installation of additional equipment. For example, we have demonstrated the feasibility of integrating electrical impedance tomography for non-invasive thermometry between the ring electrodes (CONWAY et al., 1992). 


\section{Conclusions}

It has been demonstrated that the ring applicator is capable of inducing a radiative circumferential electromagnetic field with a constructive interference pattern at the centre of the phantom. Experimentally encouraging results have been obtained with respect to the ability to perform deep heating and SAR steering in a phantom of realistic size. Of course, in a clinical situation, tissue anatomy greatly affects the SAR distribution and may cause the local maximum and the smooth profile as measured in the CDRH phantom to disappear. Current clinical experience with the second-generation radiative devices indicates a limited feasibility to adapt the SAR and temperature distribution due to tissue anatomy and blood flow.

Acknowledgments-This work was financially supported by the Dutch Cancer Society, the Danish Cancer Society and the Lund Science Award 1987.

G. C. van Rhoon wishes to express his gratitude for the kind hospitality during his stay at the Institute for Electronic Systems.

\section{References}

Conway, J., Hawley, M., Mangnall, Y., Amasha, H., and van RHoON, G. C. (1992): 'Experimental assessment of electrical impedance imaging for hyperthermia monitoring,' Clin. Phys. Physiol. Meas., Suppl. A, 13, pp. 185-189

De Leeuw, A. A. C., and Lagenduk, J. J. W. (1987): 'Design of a clinical deep-body hyperthermia system based on the "coaxial TEM' applicator,' Int. J. Hyperthermia, 3, pp. 413-421

de Leeuw, A. A. C., Lagendijk, J. J. W., and van den Berg, P. M. (1990): "SAR distribution of the "coaxial TEM" system with variable aperture width: measurements and model computations,' Int. J. Hyperthermia, 6, pp. 445-451

de Leeuw, A. A. C., Moorbroek, J., and LagendiJk, J. J. W. (1991): 'Specific absorption rate steering by patient positioning in the "Coaxial TEM" system: phantom investigation,' Int. J. Hyperthermia, 7, pp. 605-611

Feldmann, H. J., Molls, M., Adler, S., Meyer-Schwickerath, M., and $S_{A C K}, H$. (1991): 'Hyperthermia in eccentrically located pelvic tumours: excessive heating of the perineal fat and normal tissue temperatures,' Int. J. Radiat. Oncol. Biol. Phys., 20, pp. 1017-1022

Franconi, C. (1987): "Hyperthermia heating technology and devices' in: Field, S. B., and Franconi, C. (Eds.), 'Physics and technology of hyperthermia' (Martinus Nijhoff Publishers, Dordrecht, The Netherlands), pp. 80-122

Heinzl, L., Hornsleth, S. N., Raskmark, P., and Andersen, J. B. (1990): 'Electromagnetic applicators' in FIELD, S. B., and HAND, J. W. (Eds.) 'An introduction of the practical aspects of clinical hyperthermia' (Taylor \& Francis, London) pp. 275-304

KapP, D. S., Fessenden, P., Samulski, T. V., Bagshaw, M. A., Cox, R. S., Lee, E. R., Lohrbach, A. W., Meyer, J. L., and PrionAS, S. D. (1988): 'Stanford University institutional report.
Phase I evaluation of equipment for hyperthermia treatment of cancer,' Int. J. Hyperthermia, 4, pp. 75-115

Raganella, L., Banci, G., Vannucci, I., Franconi, C., and Tiberio, C. A. (1989): ' $27 \mathrm{MHz}$ conformal capacitive ring (CR) applicators for uniform hyperthermic/diathermic treatment of body segments with axial fields,' IEEE Trans., BME-36, (11), pp. $1124-1132$

Raskmark, P., and Gross, E. (1987): 'Balanced applicator tests,' Internal report, Institute for Electronic Systems, University of Aalborg, Aalborg, Denmark

SAPOzinK, M. D., GibBs, F. A., GibBs, P., and STEwart, J. R. (1988): 'Phase I evaluation of hyperthermia equipment: University of Utah Institutional Report,' Int. J. Hyperthermia, 4, pp. 117-132

SCHNEIDER, Chr., and vaN DiJK, J. D. P. (1991): 'Visualisation by a matrix of light-emitting diodes of interference effects from a radiative four-applicator hyperthermia system,' Int. J. Hyperthermia, 7, pp. 355-366

Shimm, D. S., Cetas, T. C., Oleson, J. R., Cassady, J. R., and Sim, D. A. (1988): "Clinical evaluation of hyperthermia equipment: The University of Arizona Institutional Report for the NCI Hyperthermia Equipment Evaluation Contract,' Int. $J$. Hyperthermia, 4, pp. 39-51

Sowinski, M. C., Van Putten, M. H. P. M., Van den Berg, P. M., and VAN RHOON, G. C. (1989): 'A quasi-static model for the ring capacitor applicator,' IEEE Trans., BME-36, pp. 995-1003

Stogryn, A. (1971): 'Equations for calculating the dielectric constant of saline water', IEEE Trans., MTT-19, pp. 733-736

Strohbehn, J. W., Curtis, E. H., Paulsen, K. D., Yuan, X., and LYNCH, D. R. (1989): "Optimization of the absorbed power distribution for an annular phased array hyperthermia system," Int. J. Radiat. Oncol. Biol Phys., 16, pp. 589-599

Turner, P. F., Tumeh, A., and SchaefERMeYER (1989): 'BSD-200 approach for deep local and regional hyperthermia: physics and technology,' Strahlenther. Onkol., 165, pp. 738-741

van Dijk, J. D. P., Schneider, Chr., Van Os, R. M., Blank, L. E. C. M., and Gonzalez, D. G. (1990): 'Results of deep body hyperthermia with large waveguides' in BiCHER, H. I., MCLAREN, J. R., and Pigliucci, G. M. (Eds.) 'Consensus of hyperthermia for the 1990s, Clinical practice in cancer treatment' (Plenum Press, New York) pp. 315-319

van Dujk, J. P. M., Schneider, Chr., Wust, P., Baumhoer, W., De LeEuw, A. A. C., and Olmi, R., (1992): 'Comparison of EM deep body hyperthermia systems with a matrix of LED dipoles,' COMAC-BME Hyperthermia Bull., 7, pp, 19-21

van Rhoon, G. C., Visser, A. G., VAN DEN Berg, P. M., and Reinhold, H. S. (1988): 'Evaluation of ring capacitor plates for regional deep heating,' Int. J. Hyperthermia, 4, pp. 133-142

van Rhoon, G. C., Sowinski, M. J., van den BerG, P. M., Visser, A. G., and ReINHOLD, H. S. (1990): 'A ring capacitor applicator in hyperthermia: energy distributions in a fat-muscle layered model for different ring electrode configurations,' Int. J. Radiat. Oncol. Biol. Phys., 18, pp. 77-85

Visser, A. G., van Rhoon, G. C., van den Berg, P. M., and REINHOLD, H. S. (1987): 'Evaluation of calculated temperature distributions for a $27 \mathrm{MHz}$ ridged waveguide used in localized deep hyperthermia,' Int. J. Hyperthermia, 3, pp. 245-256

Wust, P., Nadobny, J., Felix, R., Deuflhard, P., Louis, A., and JoHN, W. (1991): 'Strategies for optimized application of annular-phased-array systems in clinical hyperthermia,' Int. $J$. Hyperthermia, 7, p. 157-173 